\title{
Stratégie internationale de la France pour l'eau et l'assainissement 2020-2030, interview de Philippe Lacoste, Directeur du développement durable du ministère de L'Europe et des Affaires étrangères
}

\author{
Carole Paplorey*
}

\begin{abstract}
Résumé - En 2018, le ministère de l'Europe et des Affaires étrangères (MEAE) lançait la préparation concertée d'une nouvelle stratégie internationale en matière d'eau et d'assainissement. La concertation a pris la forme d'une consultation des parties prenantes, dont la Société hydrotechnique de France (SHF). Rapporteur, avec l'Institut de recherche pour le développement (IRD) et l'Office international de l'eau (OIEau) du collège "Recherche, formation et associations scientifiques et techniques», la SHF s'est nettement positionnée pour que les tensions sur les ressources en eau, les impacts du changement climatique, les besoins de formation et toutes les composantes d'une gestion intégrée des ressources en eau soient traités au bon niveau. La stratégie 2020-2030 ainsi élaborée a été présentée lors d'une réunion de lancement le 24 février 2020 au MEAE en présence du Secrétaire d'État Jean-Baptiste Lemoyne. Philippe Lacoste, ingénieur des travaux publics de l'État, Directeur du développement durable du MEAE nous en explique les grandes lignes. Propos recueillis par Carole Paplorey, Déléguée Générale de la SHF.
\end{abstract}

Mots clés : stratégie internationale de la France pour l'eau / aide publique au développement / services d\&apos / assainissement / eau potable / développement durable

\begin{abstract}
French international strategy for water and sanitation 2020-2030, interview of Philippe Lacoste, Director of Sustainable Development at French Ministry of Europe and Foreign Affairs. In 2018, the French Ministry of Europe and Foreign Affairs (MEAE) launched the concerted preparation of a new international water and sanitation strategy. The consultation took the form of stakeholder consultation, including la Société Hydrotechnique de France (SHF). Rapporteur, with the Research Institute for Development (IRD) and the International Office for Water (IOW) of the college "Research, training and scientific and technical associations", SHF has clearly positioned itself so that tensions on water resources, impacts of climate change, training needs and all the components of integrated water resources management are addressed at the right level. The 2020-2030 strategy thus developed was presented at a launch meeting on February 24, 2020 at the MEAE in the presence of Secretary of State Jean-Baptiste Lemoyne. Philippe Lacoste, State Public Works Engineer, Director of Sustainable Development at MEAE explains the main points. Interview by Carole Paplorey, General Delegate of SHF.
\end{abstract}

Keywords: French International strategy for water / public development aid / sanitation facilities / drinking water / sustainable development

\section{SHF : Le MEAE a lancé le 24 février dernier une stratégie internationale 2020-2030 pour l'eau et l'assainissement, quel bilan faites- vous des actions menées au cours des dernières années ?}

PL : La France est un bailleur historique dans le domaine de l'eau et de l'assainissement et elle participe activement à faire du secteur une priorité sur l'agenda européen et international.
En 1998, la Conférence internationale de Paris «Eau et Développement durable», initiative portée par la France et l'Union européenne, avait déjà permis de soutenir un programme d'actions pour l'eau et a ouvert la voie aux Objectifs du Millénaire pour le Développement (OMD), notamment l'objectif 7.C consacré à l'eau. En 2003, la France avait également placé l'eau au cœur des priorités de sa présidence du G8 et fait adopter à l'occasion du Sommet d'Evian un plan d'action dans ce domaine.

En 2005, la France a publié sa première stratégie internationale pour l'eau et l'assainissement avec pour objectif notamment le doublement de son soutien à ce secteur visant en

*Correspondance : c.paplorey@shf-hydro.org 
priorité l'Afrique. Objectif atteint puisque la part de son aide publique au développement (APD) bilatérale dans le domaine de l'eau et de l'assainissement est passée de moins de 400 millions d'euros en moyenne sur la période 2010-2013 à près de 800 millions d'euros sur la période 2014-2017. L'année 2005 a été marquée également par la promulgation de la loi OudinSantini qui a permis aux collectivités locales, agences de l'eau et syndicats d'eau de consacrer jusqu'à $1 \%$ de leur budget pour soutenir des projets de développement et de coopération en faveur des services d'eau potable et d'assainissement. Je voudrais à cet égard saluer la mémoire de M. Jacques Oudin, ancien sénateur de Vendée et co-auteur de la loi qui s'est éteint le 21 mars dernier. Cette loi pionnière a permis de mobiliser près de 300 millions d'euros entre 2007 et 2018. Cette forme de coopération décentralisée est encore peu reconnue et largement sous-utilisée. Or, elle permet non seulement de diversifier les instruments de financement mais aussi de sensibiliser les citoyens sur les actions réalisées dans le domaine de l'eau et de l'assainissement et de favoriser le partage d'expertise entre techniciens et gestionnaires de terrain en matière de développement local et de gestion des services d'eau et d'assainissement.

Partant du constat que la France et les acteurs français de l'eau devaient parler d'une seule voix pour peser plus sur la scène internationale, les pouvoirs publics ont décidé de créer, en 2007, le Partenariat français pour l'eau (PFE) regroupant l'ensemble des acteurs de l'eau: opérateurs privés et publics, élus, organisations non gouvernementales et chercheurs. Le plaidoyer du PFE, relayé par les efforts diplomatiques de la France, a activement contribué à la reconnaissance par l'Assemblée générale des Nations unies des droits à l'eau potable et à l'assainissement comme droits humains fondamentaux en 2010 , il y a tout juste 10 ans.

Après ce succès, la France a accueilli, en 2012, le Forum mondial de l'eau à Marseille. Cet évènement a permis d'identifier les priorités du secteur et de mettre la gestion de l'eau au cœur des négociations du sommet dit Rio+20. Cette conférence a été une étape clé dans la création, en 2015, d'un Objectif de Développement Durable dédié spécifiquement à la question de l'eau et l'assainissement-l'ODD numéro $6^{1}$.

\footnotetext{
${ }^{1}$ Les 17 Objectifs de Développement Durable qui ont pris la suite en 2015 des 8 Objectifs du Millénaire pour le Développement constituent le programme de développement durable à l'horizon 2030 ou Agenda 2030. Cet agenda vise la transformation de notre monde en éradiquant la pauvreté et en assurant sa transition vers un développement durable. Parmi ces 17 ODD figure l'ODD numéro 6 qui vise à «garantir l'accès de tous à l'eau et à l'assainissement et assurer une gestion durable des ressources en eau ». Au travers de ses 8 cibles, l'ambition de l'ODD 6 est ainsi d'assurer l'accès universel à l'eau potable et à l'assainissement à un coût abordable (6.1 et 6.2), d'améliorer la qualité de l'eau et réduire les pollutions (6.3), de promouvoir une utilisation efficiente et efficace des ressources en eau dans tous les secteurs - domestique, énergie, industriel, agriculture(6.4) et de préserver les écosystèmes liés à l'eau (6.6). Il cherche également à renforcer la coopération internationale et le renforcement des capacités des populations locales (6.a et 6.b) en promouvant la gestion intégrée des ressources en eau à tous les niveaux (6.5) et ce, dans un contexte de développement durable et de changements climatiques. Pour plus d'informations sur les composantes et le suivi de l'ODD 6: https://www.un.org/sustainabledevelopment/fr/waterand-sanitation/.
}

Malgré toutes les avancées auxquelles la France a contribué et les progrès enregistrés, notamment dans l'accès aux services d'eau, de grandes inégalités géographiques et économiques persistent toutefois au niveau mondial et il reste encore beaucoup à faire pour que tous les individus aient accès à ces services de base. À l'heure actuelle, selon l'ONU, près de 2,2 milliards d'individus n'ont toujours pas un accès à de l'eau potable. La gouvernance, les financements, le renforcement des capacités, et l'acquisition des données continuent d'être des enjeux de taille pour la réussite de l'ODD 6.

La France a donc une longue expérience en la matière et celle-ci repose sur la concertation et la participation des différents acteurs de l'eau. La nouvelle stratégie internationale pour l'eau et l'assainissement précise la façon dont la France entend contribuer à ses diverses problématiques pour les 10 prochaines années.

\section{SHF : Pour la période 2020-2030, sur quels grands axes le MEAE a-t-il décidé de renforcer ses engagements en matière d'eau potable?}

PL : Malgré les investissements significatifs de la France effectués au cours des dernières années, l'accès à des services d'eau potable reste un défi majeur. Chaque jour, selon l'UNICEF, environ 1000 enfants meurent de maladies d'origine hydrique telles que le choléra, le paludisme, la fièvre typhoïde ou des salmonelloses. Sans mesures supplémentaires, la qualité de l'eau continuera à se détériorer au cours des années à venir, en raison des pollutions des eaux souterraines et de surface et d'une mauvaise gestion des ressources. De plus, le changement climatique affecte d'ores et déjà fortement la disponibilité en eau dans certaines régions. Cela donne une idée de l'ampleur du défi.

Alignée sur les cibles de l'ODD 6 de l'Agenda 2030, la nouvelle stratégie internationale de la France pour l'eau et l'assainissement (2020-2030) vise deux objectifs principaux. Le premier vise l'accès universel et équitable à l'eau potable, à l'assainissement et à l'hygiène. Investir dans une eau de qualité, c'est investir pour la santé de chacun. L'accès à l'eau est fondamental pour lutter contre la propagation de maladies, notamment la pandémie de COVID-19. Rappelons que le lavage des mains à l'eau et au savon constitue le premier des gestes barrière. Le deuxième objectif vise le renforcement de la gestion intégrée des ressources en eau à l'échelle des bassins versants. Et ces bassins sont rarement limités aux frontières nationales, il s'agit donc d'encourager des modalités de gestion commune d'un bien commun.

Afin d'atteindre ces objectifs, la stratégie internationale de l'eau et de l'assainissement, qui a été lancée le 24 février 2020 par M. Jean-Baptiste Lemoyne, secrétaire d'État aux Affaires étrangères, définit 3 axes d'actions prioritaires. Tout d'abord, il s'agit d'améliorer la gouvernance du secteur de l'eau et de l'assainissement à la fois à l'échelle locale et mondiale. Pour ce faire, la France appuie la mise en œuvre de législations nationales, le renforcement des capacités des acteurs et usagers locaux. Les indicateurs de résultat sont d'ailleurs fixés sur le nombre de personnes gagnant un accès aux services d'eau gérés en toute sécurité. L'Agence française de développement (AFD) s'est fixé une cible de 4 millions de personnes, en particulier les 
plus démunis, bénéficiant d'un service d'alimentation en eau potable géré en toute sécurité. À l'international, la France encourage la gestion concertée des bassins transfrontaliers en diffusant des outils comme les Conventions d'Helsinki ${ }^{2}$ et de New York et le protocole sur l'eau et la santé. Pour une gestion équitable et durable des ressources en eau, la France favorisera également le développement de la gestion intégrée des ressources en eau afin d'encourager le dialogue dans les processus de planification et de décision et ainsi de contribuer à optimiser les usages de l'eau entre les différents secteurs d'activités.

Le deuxième grand axe de cette stratégie repose sur le renforcement de la sécurité de l'approvisionnement en eau pour tous. Face aux risques environnementaux, il s'agit d'améliorer et de diffuser les connaissances scientifiques portant sur les impacts du changement climatique sur le cycle de l'eau, tout en gérant mieux l'offre et la demande entre les différents acteurs.

Enfin, le troisième axe vise le renforcement de l'efficacité des moyens et des outils, en favorisant le développement de solutions innovantes et de mécanismes solidaires de financement. Cet engagement s'inscrit dans l'objectif du gouvernement de porter, en 2022 , l'aide au développement à $0,55 \% \mathrm{du}$ revenu national brut.

\section{SHF : Et pour l'accès aux services d'assainissement?}

PL : Aujourd'hui, quelque 4,5 milliards de personnes n'ont toujours pas accès à des services d'assainissement gérés en toute sécurité, ce qui a de graves répercussions sur la santé et le bien-être des populations ainsi que sur l'état des écosystèmes. Malheureusement, le droit à l'assainissement a longtemps été relégué au second plan derrière l'accès à l'eau potable, tant d'un point de vue politique que des financements. Les acteurs français ont œuvré pour que l'assainissement soit reconnu comme un sujet prioritaire à l'international. Et il y a urgence : selon la FAO, dans les pays en développement, environ $80 \%$ des eaux usées sont rejetées sans aucun traitement, polluant le milieu naturel et contaminant les ressources en eau.

En l'absence de pratiques d'hygiène ou d'installations, le manque d'accès à l'assainissement constitue un problème majeur de santé publique. 2,6 millions de personnes meurent chaque année en raison de maladies liées à l'eau et au manque d'assainissement selon l'OMS.

Afin de garantir l'accès équitable à l'assainissement à l'international, la nouvelle stratégie de la France prévoit d'équilibrer les financements entre eau et assainissement afin

\footnotetext{
${ }^{2}$ La Convention d'Helsinki de 1992 sur la protection et l'utilisation des cours d'eau transfrontières et des lacs internationaux, qui est entrée en vigueur en 1996, vise à prévenir et à maîtriser la pollution en assurant une utilisation rationnelle des eaux transfrontières de surface ou souterraines. Elle encourage le dialogue et une coopération internationale à travers l'échange de connaissances et d'expériences sur les différentes questions liées à l'eau. À l'heure actuelle, 43 États et l'Union européenne sont parties à la Convention. La France, cheffe de file de l'ouverture de la Convention d'Helsinki aux pays non-membres de la Commission Économique des Nations unies pour l'Europe (CEE-ONU) depuis 2013, promeut activement son adhésion à l'international (https://www.unece.org/env/water/text/text.html).
}

qu'en 2030 la moitié de l'aide publique au développement soit dédiée au secteur de l'assainissement. Au-delà des financements, la France promeut le renforcement institutionnel et juridique en faveur de l'accès à l'assainissement pour promouvoir la sensibilisation à l'hygiène, notamment l'hygiène menstruelle. Par ailleurs, la nouvelle stratégie vise la réduction de la pollution des eaux pour limiter les dégradations environnementales et les risques sanitaires potentiels. Pour protéger le cycle de l'eau, la France entend privilégier les solutions fondées sur la nature pour accroître la résilience des territoires face aux changements climatiques et sauvegarder les milieux naturels, zones humides et écosystèmes aquatiques afin de préserver les ressources. Dans le cadre de sa nouvelle stratégie, la France insiste aussi sur l'importance des travaux de recherche en particulier, interdisciplinaires, pour mettre au point des infrastructures gérées en toute sécurité, efficaces et à un coût abordable.

\section{SHF : Y a-t-il des spécificités dans l'approche française de la gestion de la ressource?}

PL : Oui, le modèle de gestion intégrée des ressources en eau ou GIRE est clairement un modèle français. Il a été mis en œuvre en France dès les années 1960. C'est un outil permettant d'utiliser durablement l'eau pour répondre aux différents besoins et notamment ceux des populations, les besoins agricoles et industriels. Cette méthode s'applique à l'échelle d'un bassin versant, qu'il soit situé à l'intérieur d'un État ou partagé entre plusieurs États. La GIRE est l'un des fondements de la coopération transfrontalière promue notamment par la Convention d'Helsinki sur la protection et l'utilisation des cours d'eau transfrontières et des lacs internationaux. En visant une conciliation de tous les usages de l'eau, cet outil permet de garantir la sécurité alimentaire, énergétique et humaine, à travers un partage équitable des ressources. Ce modèle est à présent reconnu internationalement et il constitue la cible 6.5 de l'ODD 6 de l'Agenda 2030. La France continue de défendre l'idée qu'au-delà d'une modalité de coopération, la GIRE est un instrument positif, une source de paix et de stabilisation.

La France met aussi en avant une approche par les droits ainsi que la question de l'égalité des genres dans ses interventions. C'est en effet aux femmes qu'incombe bien souvent le fardeau d'aller chercher l'eau nécessaire au foyer, ce qui limite le temps qu'elles peuvent consacrer à d'autres activités. La France entend ainsi libérer les enfants et les femmes de la corvée de l'eau et donner accès à des toilettes aux filles à l'école primaire, comme ciblé par l'ODD 4 portant sur l'accès de tous à une éducation de qualité. La France privilégie dans cette optique le financement de programmes inclusifs, en faveur des pauvres, des enfants, des femmes et des populations locales, notamment en Afrique, afin d'assurer leur participation dans les processus de décision et de gestion des ressources en eau.

Le changement climatique implique enfin un sérieux changement de paradigme et des efforts renforcés de résilience face aux nombreux risques liés à l'eau. Dans le cadre de sa nouvelle stratégie, la France vise à renforcer la gestion des risques (en particulier ceux dus à des évènements météorologiques extrêmes) et le renforcement des connaissances pour 
assurer la sécurité d'approvisionnement en eau. Pour cela, la France promeut en particulier les solutions fondées sur la nature afin d'encourager les mesures d'atténuation et d'adaptation au changement climatique mais aussi des infrastructures permettant de limiter les dégâts des crues par exemple.

\section{SHF : Comment s'organise l'aide publique au développement de la France sur la question de l'eau?}

PL: Pour atteindre l'ODD 6, l'OCDE estime qu'il est nécessaire d'y investir 1700 milliards de dollars d'ici 2030. Le principal enjeu réside dans le fait d'orienter les financements vers les pays les moins avancés et plus spécifiquement vers les 19 pays pauvres prioritaires (PPP) identifiés par le Comité interministériel de la coopération internationale et du développement (CICID). Il est également nécessaire de mieux coordonner l'action des partenaires techniques et financiers pour une meilleure efficacité de l'aide sur le terrain.

La France figure parmi les 5 plus importants bailleurs en volume aux côtés des États-Unis, de l'Allemagne, du Royaume-Uni et du Japon. Elle a consacré environ $8 \%$ de son APD nette au secteur de l'eau et de l'assainissement en 2017. Au niveau de la répartition de l'APD, plus de $80 \%$ est octroyée par le secteur public. La France vise à augmenter la part de l'APD sous forme de dons pour permettre aux pays ayant de faibles capacités à emprunter d'en bénéficier. Si l'aide bilatérale de la France a augmenté de façon significative, les montants de subventions restent encore trop faibles. La nouvelle stratégie doit permettre aux 19 PPP de notre APD de bénéficier de $50 \%$ des efforts en subvention de l'État et deux tiers des subventions mises en œuvre par l'AFD, ce qui permettra d'améliorer l'orientation géographique et sectorielle des financements. La France poursuit également ses efforts pour accélérer les décaissements des fonds, encore trop longs du fait de difficultés administratives et techniques de mise en œuvre, notamment en milieu urbain, et ainsi améliorer la transparence de l'aide. Enfin, en ce qui concerne l'efficacité de l'aide, la France vise à mieux répartir ses fonds vers des projets en zone rurale ou périurbaine, où les besoins sont les plus importants, et à prioriser également l'installation de services d'assainissement.

\section{SHF : Comment s'articulent les actions du MEAE avec le secteur privé ? Et avec les ONG?}

PL: Il est important de rappeler tout d'abord que la nouvelle stratégie internationale pour l'eau et l'assainissement résulte d'un long processus collaboratif et inclusif. L'ensemble des acteurs de l'eau a pris part à ce chantier, et notamment le secteur privé et les ONG. Ces travaux ont permis au ministère de l'Europe et des Affaires étrangères de renforcer ses partenariats et ses relations avec ces acteurs afin de mieux prendre en compte leurs réalités et dépasser les limites institutionnelles traditionnelles.

En ce qui concerne les ONG, la nouvelle stratégie prévoit d'améliorer le ciblage des financements et renforcer notamment la part d'APD transitant par les ONG. Par leur expertise sur le terrain, l'engagement de ces organisations est essentiel pour la mise en œuvre et le suivi de projets, en particulier dans le secteur humanitaire et lors d'interventions d'urgence et posturgence. En matière de sensibilisation du public et de mobilisation citoyenne, elles constituent un lien indispensable entre les populations et les institutions. Elles permettent également de faire progresser la réflexion et d'enrichir les débats sur les questions d'eau et d'assainissement par des observations de terrain qui permettent de faire évoluer les modes de faire pour une meilleure appropriation par les populations.

Les acteurs privés français de l'eau et de l'assainissement ont une influence mondiale du fait de leur grande expertise et de leur savoir-faire reconnu notamment en ce qui concerne les agglomérations urbaines qui nécessitent des infrastructures coÛteuses pour les finances publiques. Ils forment une filière d'exportation performante que le ministère de l'Europe et des Affaires étrangères promeut au titre de ses missions de diplomatie économique et nos ambassades qui suivent au titre des « grands contrats».

\section{SHF : Sur le plan du développement durable, I'ODD 6 est entièrement dédié à l'accès à l'eau potable et à l'assainissement. Comment s'inscrit cet objectif au sein du Programme de développement durable à I'horizon 2030 ?}

PL: Le manque de visibilité du secteur de l'eau et de l'assainissement faisait l'objet de nombreuses critiques vis-àvis des OMD. En effet, seule une cible, l'OMD 7.c répondait à la problématique du manque d'accès à l'eau et à l'assainissement. En tant que plan d'action de développement durable international pour « les personnes, la planète et la prospérité », l'Agenda 2030 offre une vision intégrée et holistique de l'eau et de l'assainissement et il permet ainsi de mieux appréhender les liens entre les différents ODD et les secteurs d'intervention.

Transversale par nature, la question de l'eau et de l'assainissement n'est en effet pas confinée à l'ODD 6. Dixhuit cibles concernent la gestion de l'eau et de l'assainissement directement ou indirectement, comme par exemple dans le domaine de la santé (approvisionnement en eau et à l'assainissement), de la sécurité alimentaire (irrigation des cultures), de l'énergie (production d'hydroélectricité), du développement économique (dépendance aux écosystèmes aquatiques) et de l'environnement (effets du changement climatique sur la fréquence et l'intensité des périodes d'inondation et de sécheresse). Les ambitions portées par l'ODD 6 se traduisent donc par la reconnaissance des liens entre les différents secteurs d'activités et de développement. Cet objectif favorise ainsi les synergies avec d'autres droits étroitement liés et permettent de répondre aux grands enjeux actuels de sécurité alimentaire et nutritionnelle, de santé, d'égalité entre les femmes et les hommes, de réduction des inégalités, d'éducation et de développement économique.

L'évaluation des progrès réalisés sur les ODD a aussi permis de redéfinir les statistiques sur l'accès à l'eau potable et à l'assainissement en rendant compte de critères plus précis tels que la disponibilité, la qualité, l'acceptabilité et l'accessibilité 
des services d'eau et d'assainissement. La nouvelle stratégie de la France pour l'eau et l'assainissement (2020-2030) cherche à répondre à ces objectifs ambitieux et à opérationnaliser les transformations novatrices nécessaires qu'impliquent l'accès de tous à l'eau et à l'assainissement.

\section{SHF : Les polluants d'origine agricole sont une source importante de pollution. Comment intervenir efficacement dans ce domaine?}

PL: L'utilisation de produits chimiques - pesticides et engrais azotés en particulier - et le recours à l'irrigation intensive ont permis d'accroître la production agricole permettant ainsi de répondre à la croissance démographique mondiale, la fameuse «révolution verte» qui a permis de réduire considérablement la faim par un accroissement spectaculaire de la productivité agricole. On considère aujourd'hui que $70 \%$ des prélèvements d'eau dans le monde sont consacrés aux productions agricoles. Selon la FAO, à l'horizon 2030, cette proportion devrait encore augmenter de $15 \%$. Mais les polluants d'origine agricole constituent la principale source de pollution de l'eau dans de nombreux pays et les coÛts de traitement atteindraient plusieurs milliards de dollars chaque année. La dégradation des sols et le prélèvement excessif des eaux souterraines portent atteinte à l'avenir de l'agriculture et de la sécurité alimentaire dans son ensemble. La prise de conscience des risques est aujourd'hui réelle, mais les solutions résident dans une évolution des pratiques.

Afin d'assurer une plus grande sécurité d'approvisionnement tant d'un point de vue quantitatif que qualitatif, il est en particulier souhaitable de limiter les consommations de l'agriculture en eau, notamment en sensibilisant à des usages sobres en eau qui ne sont pas toujours très coÛteux (la technique du goutte à goutte en est un bon exemple) ou qui faisaient partie des connaissances dites traditionnelles balayées par l'industrialisation. Il est également nécessaire de transformer certains modes de production et d'adopter des pratiques plus durables telles que l'agro-écologie, qui limitent l'utilisation de produits phytosanitaires. Transformer le secteur agricole requiert de changer la manière dont nous pensons et nous consommons l'eau. Il me semble que la demande sociale s'accroît dans ce domaine et rejoint une équation aujourd'hui insoluble aux taux de progression actuels.

\section{SHF : Les facteurs de tension sur l'eau aboutissent à des situations d'inégalité et de conflits dans le monde. Quels sont les principaux enjeux humains et politiques aujourd'hui?}

PL : Il suffit de prendre un planisphère pour remarquer que les ressources en eau sont inégalement réparties sur Terre. La géographie de l'eau est une problématique de premier plan, dans un monde où $60 \%$ des ressources naturelles renouvelables en eau douce sont situées dans 9 pays. On compte aujourd'hui 263 bassins internationaux qui hébergent $40 \%$ de la population mondiale. De nombreux États sont ainsi interdépendants de cette ressource.

L'un des défis majeurs de ces prochaines années est lié aux changements climatiques qui modifient considérablement la répartition des ressources et le cycle de l'eau. Avec plus de $90 \%$ des catastrophes naturelles liées à l'eau, les changements climatiques influencent directement la disponibilité, la quantité et la qualité des ressources hydriques. Accentuée par une forte pression démographique et une urbanisation croissante, la situation se détériore et les ressources en eau deviennent de plus en plus convoitées. De nombreux pays vont être affectés par ces changements et en particulier les géographies déjà confrontées au manque d'eau comme le Sahel. Ce déséquilibre entre disponibilité et demande provoque des tensions importantes entre usagers de l'eau tant sur le périmètre national qu'entre États riverains. L'eau devient dans ces régions un catalyseur de conflits nuisibles à la paix et à la stabilité politique et sociale des pays et à leur développement. D'après l'ONU, $40 \%$ des conflits qui ont éclaté au cours des 60 dernières années seraient liés d'une façon ou d'une autre aux ressources naturelles, dont l'eau. À cet égard, il est important de rappeler la nécessité, je le répète, de promouvoir les Conventions d'Helsinki et de New York pour guider toute l'action de la communauté internationale sur la protection et l'utilisation des cours d'eau transfrontaliers et des lacs internationaux. Dans d'autres cas, il ne s'agit pas d'un problème de manque mais plutôt de gestion de la ressource. De nombreux cas de pollution, dus souvent à des accidents industriels ayant entraîné la pollution d'un cours d'eau, ont provoqué de fortes tensions diplomatiques. La construction de barrages souvent à des fins de production d'énergie électrique renouvelable est aussi l'origine d'inquiétudes ouvertes pour les États de l'aval.

Cette crise de l'eau continuera ainsi de nourrir les inégalités existantes et en créera de nouvelles en matière d'accès aux services d'approvisionnement de la ressource en particulier pour les femmes, enfants et populations marginalisées notamment en zones rurales. Ceci est d'autant plus grave que l'on constate une intensification de la concurrence entre les différentes catégories d'usagers (agriculture, ménages, industrie ou énergie) et des conflits interrégionaux potentiels. Or, les pays pauvres ou en développement dépendent beaucoup plus des activités agricoles que les autres, et l'agriculture constitue le secteur le plus consommateur d'eau, comme évoqué précédemment. L'avenir de notre écosystème commun et de notre adaptation au changement climatique dépendra donc de la manière dont nous résoudrons les défis de l'eau. Si rien n'est fait pour enrayer cette situation, d'ici 2050, 40\% de la population mondiale sera confrontée à des graves pénuries d'eau provoquant des régressions des PIB nationaux, un accroissement de la pauvreté, et une accélération des mouvements migratoires.

\section{SHF : En matière de gouvernance sur l'eau, comment renforcer une action internationale concertée?}

PL : Le secteur de l'eau et de l'assainissement dépend d'une gouvernance à la fois complexe, fragmentée et interdépendante où les acteurs sont nombreux, privés comme publics, et 
interviennent à différentes échelles, à divers niveaux de responsabilités et dans des domaines variés. Or, seule une gouvernance concertée, coordonnée, et ouvrant la voie au dialogue, permettra de répondre aux nombreux défis environnementaux, humains, sociaux et économiques qui menacent le secteur.

À cet effet, aux côtés de l'Allemagne, des Pays-Bas et de la Suisse, la France plaide activement pour le renforcement d'ONU-Eau, bureau de coordination des activités et des organismes du système onusien traitant des questions liées à l'eau et à l'assainissement et des progrès de l'ODD 6, en le mandatant pour l'organisation d'évènements onusiens, le suivi $\mathrm{du}$ secteur, et la formulation de recommandations pour l'atteinte des cibles des ODD.

Il apparaît également de plus en plus clairement qu'il est souhaitable d'adopter une gestion intégrée et durable des ressources en eau et de délimiter qui fait quoi, pourquoi et comment. Les usages de l'eau relèvent d'activités différentes qui obéissent chacun à leur logique et peuvent nourrir des conflits latents, nous l'avons déjà abordé. Aujourd'hui, face au défi de l'eau, il convient d'aller au-delà des questions de financements et d'infrastructures, et promouvoir la coopération entre pays et usagers pour partager les connaissances et les ressources. C'est pourquoi la France s'est positionnée comme cheffe de file de l'ouverture de la Convention d'Helsinki sur la protection et l'utilisation des cours d'eaux transfrontières et des lacs internationaux adoptée en 1992, ouverture obtenue en 2013. Elle promeut activement l'adhésion des pays, en particulier en Afrique où coulent de grands fleuves traversant plusieurs pays. À ce jour, le Sénégal et le Tchad l'ont ratifiée. D'autres États devraient les rejoindre sous peu renforçant ainsi les autorités internationales de bassin déjà existantes.

\section{SHF : Enfin, plus globalement sur la stratégie 2020-2030 du MEAE, comment seront évalués les progrès réalisés pour l'accès à l'eau potable et aux services d'assainissement?}

PL: Afin d'en assurer le suivi, cette nouvelle stratégie intègre un cadre de redevabilité qui regroupe 17 indicateurs associés aux différents axes stratégiques. Pour éviter la multiplication des indicateurs et des méthodologies, cette matrice se base sur les critères d'évaluation des projets du Comité d'aide au développement de l'OCDE, des Nations unies, de l'OIEau, du Programme Solidarité Eau, et de l'Agence française de développement. Pour assurer un alignement optimal entre les objectifs de la stratégie dont nous parlons ici, les activités qui auront été entreprises, et les orientations politiques du Comité interministériel de la coopération internationale et du développement, trois phases sont distinguées.

Tout d'abord, la stratégie fera l'objet d'une évaluation annuelle à la fois quantitative et qualitative. Celle-ci permettra de mettre en lumière chaque année les progrès réalisés en matière d'accès aux services d'eau, d'assainissement et d'hygiène. À cet objectif s'ajoute celui d'assurer un suivi du modèle de gestion intégrée des ressources en eau et d'améliorer la gouvernance du secteur. L'approche intégrée et dirigée vers les besoins des usagers étant privilégiée, une attention particulière sera apportée à l'appropriation par les différentes parties prenantes, en particulier les populations locales, afin qu'elles puissent être associées aux prises de décisions en toute connaissance de cause. Ceci se traduit également par le renforcement des dispositifs de sensibilisation et de formation aux pratiques d'hygiène (le WASH dans la terminologie des Nations unies). Un suivi spécifique sera effectué pour les 19 pays prioritaires visés par l'APD française dans le but d'orienter les financements, en particulier ceux de l'AFD et des États membres de l'OCDE, en faveur du secteur considéré.

Dans un second temps, une revue à mi-parcours est prévue en 2025 pour que soient observées les inflexions des politiques et surtout de la mise en œuvre des projets et programmes en lien avec l'Agenda 2030. Ce cadre de suivi doit ainsi permettre lévaluation des actions de la France sur une base transparente et objective, ce qui est nécessaire pour renforcer son efficacité et aussi assurer une gestion cohérente de nos moyens au service du développement durable au plan international compte tenu de nos priorités sectorielles et géographiques.

Enfin, un bilan de la stratégie à son terme en 2030 sera réalisé pour continuer à impulser une action innovante et soutenable, en termes de gouvernance comme de mise en œuvre sur le terrain. Tout cela est ambitieux j'en conviens mais le secteur de l'eau et de l'assainissement, fondement de toute vie, le mérite.

Citation de l'article : Paplorey C. 2020. Stratégie internationale de la France pour l'eau et l'assainissement 2020-2030, interview de Philippe Lacoste, Directeur du développement durable du ministère de L'Europe et des Affaires étrangères. La Houille Blanche : 101-106 